\title{
Enfermagem e educação em saúde de hipertensos: revisão da literatura
}

\author{
Nursing and health education in hypertensive patients: \\ literature review
}

\section{André Almeida de Moura ${ }^{1}$ Maria Suely Nogueira ${ }^{2}$}

\section{Resumo}

A hipertensão arterial é um dos grandes problemas de saúde pública no país e no mundo. A educação em saúde caracteriza-se em uma estratégia importante para sucesso do tratamento quer seja medicamentoso ou não, dos hipertensos. Este estudo busca conhecer o uso da Educação em Saúde como prática no cotidiano do Enfermeiro para o enfrentamento da Hipertensão Arterial. O estudo constituiu-se de pesquisa bibliográfica na base de dados Literatura Latino-Americana e do Caribe em Ciências da Saúde (LILACS). Para tanto, utilizou-se os seguintes descritores de assunto: Educação em saúde, enfermagem e hipertensão totalizando 46 artigos iniciais e mediante aos critérios de inclusão e exclusão restaram apenas 20 analisados. A maioria dos trabalhos foi produzida depois de 2006, há predomínio de abordagem descritiva, sendo São Paulo e Ceará, os dois estados com maior produção. A relação entre os descritores apontam para concentração de trabalhos com temáticas voltadas para a adesão ao tratamento e educação em saúde de uma forma emancipatória.

\footnotetext{
Abstraet

Hypertension is a major public health problem in the country and the world. Health education is characterized in an important strategy for successful treatment whether medical or otherwise, of hypertension. This study seeks to understand the use of the Health Education as in daily practice nurse to face the Hypertension. The study consisted of a literature database on Latin American and Caribbean Health Sciences (LILACS). Most of the work was produced after 2006, were mainly descriptive approach, and are the two states with the highest production São Paulo and Ceará. The relationship between the descriptors indicate concentration of work with themes focused on treatment adherence and health education in an emancipatory.
}

Descritores: Educação em saúde; Hipertensão; Enfermagem.

Keywords: Health Education; Hypertension; Nursing.
1 Mestrando do Programa de Enfermagem Fundamental da Escola de Enfermagem de Ribeirão Preto EERP/USP

2 Professora Doutora Associado do Departamento de Enfermagem Geral - Especializada da Eseola de Enfermagem de Rỉbeirão Preto da Universidade de São Paulo 
Introdựão

A hipertensão arterial sistêmica (HAS) é a mais frequente das doenças cardiovasculares. É considerada o principal fator de risco para várias doenças como o infarto agudo do miocárdio, acidente vascular cerebral, doença renal crônica, além da doença arterial periférica. Estima-se que no Brasil cerca de 17 milhões de indivíduos sejam portadores de hipertensão arterial, correspondendo a 35\% da população com idade de 40 anos ou mais. Este número é crescente, e, além disso, está aumentando o número de crianças e adolescentes com a doença ${ }^{1}$.

"A epidemiologia da hipertensão arterial no Brasil apresenta aspectos peculiares e coincidentes com outros países com impacto importante no perfil de mortalidade, tais como desigualdade social na distribuição do risco de morte, no acesso ao diagnóstico de hipertensão arterial e na proporção de indivíduos com pressão arterial elevada²."

As mudanças sociais, econômicas e demográficas através da transição epidemiológica no Brasil contribuíram para o acréscimo considerável da morbimortalidade por doenças crônicas nãotransmissíveis, entre as quais a HAS é a maior representante. Dessa maneira, o conceito de hipertensão arterial passou a ser analisado pela estratificação do risco cardiovascular e não somente pelos níveis pressóricos, ou seja, hipertensão não pode ser compreendida apenas como uma condição clínica de cifras tensionais elevadas, mas deverá ser levado em conta sua sintomatologia, incluindo alterações hemodinâmicas, tróficas e metabólicas ${ }^{3-4}$.

Por ser assintomática na maior parte do seu curso, a HAS tem seu tratamento negligenciado, somando-se a baixa adesão ao tratamento por parte do paciente ${ }^{1,5,6}$.

Importante ressaltar que o tratamento da hipertensão arterial não envolve apenas a utilização de medicamentos é preciso também investir na modificação do estilo de vida7. Sendo assim, faz-se necessário mudanças em alguns hábitos e costumes, em relação à alimentação, ao uso de tabaco e de bebida alcoólica, bem como a prática de exercícios físicos. Mudar o hábito de vida envolve mudanças na forma de viver e na própria ideia de saúde que o indivíduo possui ${ }^{8}$.

Mudar o estilo de vida é uma tarefa difícil, e na maioria das vezes é acompanhada de muita resistência, por isso, a maioria das pessoas não consegue fazer modificações e, especialmente, mantêlas por muito tempo, neste contexto se insere os aspectos relacionados à adesão.
A questão da adesão ao tratamento é bastante complexa e, mesmo conhecendo os fatores envolvidos, jamais será possível controlála. Entretanto, essa realidade não pode impedir que os profissionais de saúde deixem de atentar-se aos pacientes, uma vez que estes só conseguirão os tratamentos necessários quando puderem reformular ou adquirir os novos papéis, que sua condição atual de saúde exigir e assim, ter interferência na sua qualidade de vida e sua maneira de ser' .

A HAS acarreta transformações significativas na vida dos indivíduos, sejam elas na esfera psicológica, familiar, social ou econômica pela possibilidade de agravo em longo prazo. Portanto não somente o paciente como seus familiares, amigos e profissionais de saúde devem ajudar na mudança dos hábitos ${ }^{10}$.

Sendo assim, a relação dos membros da equipe de saúde com o paciente hipertenso, é um fator altamente interveniente no processo do tratamento. Deve-se aperfeiçoar recursos e estratégias junto aos hipertensos, com a participação ativa dos mesmos, visando a manutenção da qualidade de vida. O estabelecimento de uma relação aberta com o hipertenso bem como a identificação de seu conhecimento e as suas preferências, permite uma maior adesão ao tratamento ${ }^{11}$.

A educação em saúde é um campo amplo, para a qual concentram diversas concepções, tanto da área da educação, quanto da área da saúde, as quais refletem diferentes apreensões do mundo, definidas por distintas posições político-filosóficas sobre o homem e a sociedade ${ }^{12}$.

A educação em saúde constitui-se em uma ferramenta que os profissionais de saúde, entre eles os enfermeiros, devem adotar com vistas ao atendimento integral do individuo portador de hipertensão arterial. Por meio da educação em saúde, pode se gerar oportunidades de reflexão sobre saúde, práticas de cuidados e mudanças de costumes, constituindo-se um dos pilares da promoção da saúde.

O papel de educador da equipe multiprofissional, que desenvolve ações junto a portadores de hipertensão arterial, deve orientar o hipertenso para o autocuidado com vistas a diminuir as taxas de não-adesão ao tratamento ${ }^{13}$.

Ressalta-se, entretanto, que para a efetividade das ações de educação em saúde é preciso repensar a comunicação desenvolvida entre o profissional e o paciente, pois esta não 
deve constituir-se apenas em um instrumento do cuidar, mas sim, em um denominador comum das ações de enfermagem, independentemente da função que ocupa ${ }^{14}$.

Neste contexto e partindo do pressuposto que o Programa Saúde da Família (PSF) constitui-se num modelo de assistência voltado para ações preventivas e de promoção da saúde dos indivíduos, famílias e comunidades, faz-se necessário que as ações aí desenvolvidas estejam em consonância com os preceitos da educação em saúde. Neste sentido as ações educativas desenvolvidas no âmbito do PSF precisam ser reorganizadas com foco no modelo assistencial, pautado no fortalecimento da atenção à saúde, com ênfase na integralidade da assistência, no tratamento do indivíduo como parte integrada à família, ao domicílio e comunidade ${ }^{15-16}$.

Destaca-se ainda que o processo de educação em saúde possibilita um crescimento de ambas as partes, quer seja dos pacientes e também dos profissionais,além do engrandecimento pessoal e humano. Muitas vezes os pacientes demonstram uma carência de diálogo e atenção nessa troca de conhecimento $^{17}$.

Contudo, este estudo buscar conhecer o uso da Educação em Saúde como prática no cotidiano do Enfermeiro para o enfrentamento da Hipertensão Arterial, a partir do levantamento bibliográfico no LILACS, tendo como norteadora as seguintes questões: qual tem sido a produção científica nacional a respeito da educação em saúde em hipertensão arterial desenvolvidos pela enfermagem? Que características metodológicas e concepções compõem os estudos nesta temática?

\section{Pereurso metodológieo}

O estudo constituiu-se de pesquisa bibliográfica dirigida para trabalhos que apresentavam propostas ou relatos de experiências educativas com usuários hipertensos, publicados no período de 1990 até 2010. Foram analisados apenas artigos de periódicos nacionais, excluindo-se livros, teses, conferências e outros, pela dificuldade de acesso.

Para a busca dos artigos foram utilizadas as bases de dados da Literatura Latino-Americana e do Caribe em Ciências da Saúde (LILACS) através da Biblioteca Virtual em Saúde (BVS) e acesso via internet. Os descritores foram selecionados a partir das definições encontradas nos Descritores em Ciência da Saúde (DeCS), sendo eles: Hipertensão,
Educação em Saúde e Enfermagem para a busca na base de dados.

Após a leitura de resumos e dos artigos, ao total, foram encontrados 46 estudos, tendo sido excluídos alguns trabalhos por constituir - se em teses, dissertações, monografias, não estarem relacionados com a temática.

\begin{tabular}{|l|l|c|c|c|}
\hline Fonte & Descritores & Encontrados & Excluídos & Selecionados \\
\hline LILACS & $\begin{array}{l}\text { Hipertensão, } \\
\text { Educação } \\
\text { em Saúde e } \\
\text { enfermagem }\end{array}$ & 46 & 26 & 20 \\
\hline & Total & $\mathbf{4 6}$ & $\mathbf{2 6}$ & $\mathbf{2 0}$ \\
\hline
\end{tabular}

Fonte: Pesquisa Direta

Quadro 1. Levantamento bibliográfico na base de dados LILACS

\section{Resultadlos e Diseussão}

Da pesquisa bibliográfica efetuada obteve um total 46 artigos publicados entre os anos de 1990a 2010. Após leitura, selecionou-se 20 trabalhos, iniciando leituras exaustivas dos textos, fazendo uma síntese de cada artigo e categorizando os tipos de ações educativas e outros aspectos importantes. A distribuição do número de publicações pelo ano de publicação está disposta na tabela 1 .

\begin{tabular}{|c|c|c|}
\hline Ano de publicação & $\mathrm{N}$ & $\%$ \\
\hline 2010 & 02 & 10 \\
\hline 2009 & 01 & 05 \\
\hline 2008 & 03 & 15 \\
\hline 2007 & 04 & 20 \\
\hline 2006 & 04 & 20 \\
\hline 2005 & 01 & 05 \\
\hline 2004 & 01 & 05 \\
\hline 2003 & 01 & 05 \\
\hline 1995 & 01 & 05 \\
\hline 1994 & 01 & 05 \\
\hline 1992 & 01 & 05 \\
\hline Total & 20 & 100 \\
\hline
\end{tabular}

Fonte: Pesquisa Direta

Tabela 1. Distribuição dos artigos segundo período de publicação

Os dados mostram um aumento do numero de estudos a partir do ano de 2006, correspondendo a mais de $70 \%$ da produção encontrada. Este achado vai ao encontro dos 
resultados de uma pesquisa, em que os autores apontam que a produção acerca de processos educativos em saúde cresceu a partir da década de 90 e atribuem tal acréscimo à necessária revisão do modelo assistencial e a implantação do Programa de Saúde da Família como no caso do Brasil15.

\begin{tabular}{|c|c|c|}
\hline Revista/periódico & $\mathrm{N}$ & $\%$ \\
\hline Revista Brasileira de Enfermagem & 04 & 20 \\
\hline Online Brazilian Journal of Nursing & 03 & 15 \\
\hline Revista Enfermagem UERJ & 02 & 10 \\
\hline Revista Nursing & 02 & 10 \\
\hline Revista Latino-americana de Enfermagem & 02 & 10 \\
\hline Revista Texto \& Contexto & 02 & 10 \\
\hline Revista Mineira de Enfermagem & 01 & 05 \\
\hline Revista Paulista de Enfermagem & 01 & 05 \\
\hline Revista Baiana de Saúde Pública & 01 & 05 \\
\hline Revista Escola de Enfermagem USP & 01 & 05 \\
\hline Arquivos Ciências Médicas & 01 & 05 \\
\hline Total & 20 & 100 \\
\hline
\end{tabular}

Fonte: Pesquisa Direta

Tabela 2. Distribuição dos artigos segundo a fonte de publicação.

Nesta tabela ficou evidente a diversidade de periódicos em que a temática foi publicada. Acrescenta-se a isso, o crescimento da produção científica em enfermagem e também o reflexo sobre como a profissão tem utilizado do conhecimento produzido nas suas ações.

\begin{tabular}{lcc}
\hline Tipo de estudo & $\mathrm{N}$ & $\%$ \\
\hline Descritivo-exploratório & 12 & 60 \\
Qualitativo & 03 & 15 \\
Caso-controle (Intervenção) & 01 & 05 \\
Revisão bibliográfica/literatura & 03 & 15 \\
Relato de experiência & 01 & 05 \\
\hline Total & 20 & 100 \\
\hline Pesquisa Direta &
\end{tabular}

Tabela 3. Distribuição dos artigos de acordo com o delineamento do estudo

Evidencia-se que a maioria dos artigos utilizou da metodologia descritiva, entretanto foi possível encontrar outros métodos de investigação como a pesquisa qualitativa, a revisão bibliográfica, o relato de experiência e o caso-controle.

Seguindo nessa perspectiva, cabe ressaltar que os estudos exploratórios permitem ao pesquisador aumentar sua experiência sobre um determinado problema, descrevendo os fatos e fenômenos conforme a realidade ${ }^{18}$.

A pluralidade de delineamentos existentes constitui-se de ferramentas que a enfermagem pode utilizar para buscar respostas aos seus questionamentos.

Importante ressaltar que em suas experiências com o cuidar, os enfermeiros encontrem objetos para suas investigações e busquem explorar estas dimensões com vistas a melhor compreendê-las, prevê-las e controlá-las. Portanto, tanto estudos descritivos com abordagem quantitativa, os estudos qualitativos, quanto estudos experimentais, tem contribuído para o caráter científico da enfermagem ${ }^{19}$.

\begin{tabular}{|l|l|l|l|l|}
\hline Estado & $\mathrm{N}$ & $\%$ & Instituições & $\mathrm{N}$ \\
\hline Bahia & 01 & 05 & UFBA & 01 \\
\hline \multirow{2}{*}{ Ceará } & 07 & 35 & UECE & 03 \\
\cline { 4 - 5 } & & & UNIFOR & 03 \\
\cline { 4 - 5 } & & & UFC & 01 \\
\hline Goiás & 01 & 05 & UFG & 01 \\
\hline Mato Grosso do Sul & 01 & 05 & UCDB & 01 \\
\hline Minas Gerais & 02 & 10 & UFMG & 01 \\
\cline { 4 - 5 } & & & UNIFENAS & 01 \\
\hline Paraná & 01 & 05 & UFPR & 01 \\
\hline Rio de Janeiro & 02 & 10 & UFF & 01 \\
\cline { 4 - 5 } & & & FESS & 01 \\
\hline São Paulo & \multirow{2}{*}{25} & USP & 02 \\
\cline { 4 - 5 } & & & FAMERP & 02 \\
\cline { 4 - 5 } & & & INCOR & 01 \\
\hline Total & 20 & 100 & - & 20 \\
\hline
\end{tabular}

Fonte: Pesquisa Direta

Quadro 2. Distribuição dos artigos pelo estado onde foi realizado o estudo e as instituições vinculadas

O Quadro 2 mostra que algumas instituições com experiência consolidada em pesquisa já desenvolvem estudos sobre a temática há algum tempo, razão que pode justificar o aumento do numero de artigos. Tal fato ocorre pelas instituições possuírem Ligas ou Centros de Estudos relacionados com a Hipertensão Arterial. Outro fato importante a destacar é que não há semelhante distribuição de estudos por regiões do país, ausência de artigos vinculados a instituições da região norte.

Acrescenta-se a este aspecto que os cursos de pós-graduação deram efetivo impulso à produção científica na enfermagem, permitindo um avanço na avaliação crítica da prática profissional. E que a expansão dos cursos de pósgraduação no país, principalmente, em regiões em 
que não contam com esse nível de ensino ainda é um grande desafio ${ }^{20}$.

Como a hipertensão arterial é uma doença crônica e de grande importância epidemiológica no Brasil, os estudos voltam-se, dessa forma para a questão da adesão ao tratamento da doença, correspondendo há quase $50 \%$ dos artigos identificados. Outro aspecto importante evidenciado é a questão da educação em saúde de forma emancipatória aos hipertensos, visto que os profissionais de enfermagem devem despertar o senso crítico dos seus pacientes a fim de conscientizá-los da importância da doença em suas vidas e, as implicações desta quanto ao não seguimento da terapêutica estabelecida.

A hipertensão arterial é uma doença crônica e de grande importância epidemiológica no Brasil, grande parte dos estudos encontrados relacionam a hipertensão arterial e educação em saúde a fim de promover maior adesão ao tratamento.

Outro aspecto importante evidenciado é a questão da educação em saúde de forma emancipatória aos hipertensos, visto que os profissionais de enfermagem devem despertar o senso crítico dos seus pacientes a fim de conscientizá-los da importância da doença em suas vidas e, as implicações desta quanto ao não seguimento da terapêutica estabelecida. A produção de conhecimento de enfermagem tem demonstrado que o princípio de conhecimento-emancipação é cada vez mais incorporado em sua prática ${ }^{21}$.

A produção de estudos destaca, também, a educação em saúde como ferramenta de ação da equipe multiprofissional, do processo de enfermagem, além de outros aspectos relacionados.

Neste sentido, vale a pena registrar que há uma tendência, especialmente entre enfermeiros, na busca de estratégias que melhor fundamentem sua prática e que possam contribuir para a melhor assistência aos pacientes.

\section{Conelusões}

O desenvolvimento do presente estudo possibilitou verificar que a enfermagem tem buscado desenvolver trabalhos correlacionando a educação em saúde e a hipertensão arterial, especialmente em centros de estudos como São José do Rio Preto e Fortaleza. O método utilizado é diverso, o que possibilita diversas visões sobre o mesmo tema.

A maioria das pesquisas desenvolvidas enfoca a educação em saúde como estratégia para promover a adesão do paciente ao tratamento, também utilizam da educação emancipatória, que permite ao indivíduo hipertenso não ser apenas mero receptor de informações, mas que seja despertado para o autocuidado e mudanças no seu estilo de vida.

Neste contexto, fica evidente que a prática de educação em saúde deve constituir-se em parte integrante da profissão de enfermagem, muito embora, ainda sejam poucos os estudos que tratam da temática ${ }^{20}$.

Da análise mais ampla sobre os artigos pode-se identificar que os mesmos centralizam seus objetos de estudo, na maioria das vezes, no paciente, sua resposta ao tratamento, ou então, analisam o tipo de educação empregada pelos profissionais, permitindo identificar lacuna de estudos no que se refere à visão dos profissionais de enfermagem sobre a temática.

A abordagem do tema educação em saúde deve possibilitar aos envolvidos no processo o conhecimento dos limites e possibilidades de cada um, especialmente em se tratando de educação em saúde em portadores de doença crônica. Portanto, identificar quais são as dificuldades daqueles que desenvolvem educação em saúde, seja individualmente ou em grupo, ainda é um desafio.

\section{Referências}

1. Brasil. Secretaria de Atenção à Saúde, Diretoria da Atenção Básica, Ministério da Saúde. Cadernos de Atenção Básica: hipertensão arterial sistêmica para o SUS. Brasília (Brasil): Ministério da Saúde.2006. 58 p.

2. Lotufo PA. Epidemiologia da hipertensão arterial no Brasil. In: Tratado de Cardiologia SOCESP. Barueri: Manole; 2005. p. 395- 404.

3. Chaves ES, Lúcio IML, Araújo TL, Damasceno, MMC. Eficácia de programas para adultos portadores de hipertensão arterial. Rev. Bras. Enferm. 2006; 59 (4): 543-47.

4. Gasques JCP, Roland DMS, Cesarino CB. Caracterização da crise hipertensiva em pacientes de grupos de hipertensão de um ambulatório-escola. REnferm UERJ. 2008; 16 (1): 46-50.

5. Sociedade Brasileira de Hipertensão Arterial. V Diretrizes Brasileiras de Hipertensão Arterial.RevBras Hipertensão. 2006; 13(4): 256-312.

6. Plavnik FL, Colombo. Avaliação do paciente hipertenso. In: Tratado de Cardiologia SOCESP. Barueri: Manole; 2005. p. 436- 445.

7. Péres DS, Magna JM, Viana LA. Portador da hipertensão arterial: atitudes, crenças, percepções, pensamentos e práticas. Rev. Saúde Pública. 2003; 37(5): 635-42.

8. Jardim PCBV, Souza ALL, Monego ET. Atendimento multiprofissional ao paciente hipertenso. Medicina. 1996; 29: 232-38. 
9. Lessa I. Não adesão ao tratamento da hipertensão arterial: consequências econômicas para o indivíduo e para a sociedade. In: Nobre F, Pierin AMG, Mion Júnior D. Adesão ao tratamento - o grande desafio da hipertensão. São Paulo: Lemos; 2001. p. 86 -106.

10. Bastos DS, Borenstein MS. Identificando os déficits de autocuidado de clientes hipertensos de um centro municipal de saúde. Rev. Texto Contexto Enferm. 2004; 13(1) : 92-99.

11. Pierin MG, Mion Junior D. Atuação da equipe de enfermagem na hipertensão arterial. In: Instituto para o desenvolvimento da Saúde, USP, Ministério da Saúde. Manual de Enfermagem: Programa de Saúde da Família. Brasília.2001; p. 149-54.

12. Schall VT, Struchiner M. Educação em saúde: novas perspectivas. Cad. Saúde Pública. 1994; 15 Suppl2 :4-6.

13. Figueiredo JS. Desafios e perpesctivas em atividades educativas de promoção da saúde de um grupo de portadores de hipertensão arterial sob paradigma da interdisciplinaridade [thesis]. Ribeirão Preto: Escola de Enfermagem/USP; 2006. 331 p.

14. Azevedo RCS. A comunicação como instrumento do processo do cuidar: visão do aluno de graduação. Rev. Nursing. 2004; 45 (5):19-23.

15. Toledo MM, Rodrigues SC, Chiesa AM. Educação em saúde no enfrentamento da Hipertensão arterial: uma nova ótica para um velho problema. Rev. Texto ContextoEnferm. 2007; 16(2):233-38.

16. Chiesa AM, Veríssimo MDLOR. Educação em saúde na prática do PSF. In: Instituto para o desenvolvimento da Saúde, USP, Ministério da Saúde. Manual de Enfermagem: Programa de Saúde da Família. Brasília.2001; p. 34-42.

17. Moura AA, Silva DC, Biffi EFA. Relato de experiência sobre o projeto Lar de Veneranda - contribuindo para a formação do enfermeiro. Revista de Educação Popular.1 (5):53-56.

18. Trivinos ANS. Introdução à pesquisa em ciências agrárias - a pesquisa qualitativa em educação. São Paulo: Atlas. 1994

19. Guedes MVC, Silva LF, Freitas MC. Educação em saúde: objetivo de estudo em dissertações e teses de enfermeiras no Brasil. Rev. Bras. Enferm. 2004; 57(6): $662-65$.

20. Santos TCF, Gomes MLB. Nexos entre pós-graduação e pesquisa em Enfermagem no Brasil. Rev. Bras. Enferm. Brasília 2007 jan-fev; 60(16):91-5.

21. Acioli S. A prática educativa como expressão do cuidado em saúde pública. Rev. Bras. Enferm. 2008; 61 (1):117-21. 